\title{
belarus-
}

analysen

\section{LITERATUR-NOBELPREIS FÜR SWETLANA ALEXIJEWITSCH}

- ANALYSE

Literatur-Nobelpreis für Swetlana Alexijewitsch

2

Von Yvonne Pörzgen, Bremen

ANALYSE

Belarussische Dilemmata. Die Rezeption des Nobelpreises für Swetlana Alexijewitsch in Belarus Von Felix Ackermann, Vilnius

UMFRAGE

Die Bekanntheit von Swetlana Alexijewitsch in der russischen Bevölkerung

CHRONIK

12. Oktober - 29. November 2015 


\section{Literatur-Nobelpreis für Swetlana Alexijewitsch}

Von Yvonne Pörzgen, Bremen

\section{Ist das Literatur?}

"Ist das denn Literatur?« Diese Frage haben sich so manche gestellt, als am 8. Oktober 2015 bekannt wurde: Swetlana Alexijewitsch ist die diesjährige Preisträgerin des Literaturnobelpreises. Alexijewitsch erzählt kaum, sondern lässt erzählen. Der Literaturkritiker Denis Scheck bezeichnete Alexijewitsch als "Jägerin des verlorenen O-Tons«, von sich selbst sagt die Autorin, sie schreibe im "Genre der Stimmen«. Die Autorin gibt in ihren "zeithistorischen Collagen«, wie der Historiker Jan C. Behrends Alexijewitschs Bücher bezeichnet, den Stimmen anderer Raum. Durch die Verdichtung und Komposition ihres Quellenmaterials, auf die Behrends mit Recht verweist, ist Alexijewitsch mehr als "die großartige Publizistin«, als die Iris Radisch sie im Feuilleton der "Zeit« betitelte. Diese Verfahren stellen gerade die "genuin schöpferische Leistung« dar, die Radisch bei Alexijewitsch nicht sehen mag. Für ihre literarischen Arbeiten greift Alexijewitsch auf Techniken zurück, die sie in ihrem Journalistikstudium in Minsk und während ihrer Arbeit für Zeitungen und Zeitschriften erlernt und erprobt hat.

Geboren wurde Swetlana Alexijewitsch 1948 im ukrainischen Iwano-Frankiwsk. Ihre Mutter war Ukrainerin, ihr Vater Belarusse. Alexijewitschs Sprache war immer das Russische. Daran änderten auch die mehr als zehn unter anderem in Deutschland und Italien verbrachten Jahre nichts. Seit 2011 lebt sie wieder in Minsk.

\section{Die ganz großen Themen}

Alexijewitsch widmet sich unbequemen Themen der sowjetischen Kriegs- und Nachkriegszeit und der Zeit nach dem Zerfall der Sowjetunion. Dafür wählt sie die Perspektive des einzelnen und macht deutlich, wie die großen Entwicklungen und Entscheidungen sich auf der individuellen Ebene auswirken.

1985 erschien Alexijewitschs erstes Buch »Der Krieg hat kein weibliches Gesicht" (U wojny - ne zhenskoje lizo, dt. 1987). Sie verfolgte darin das Ziel, das allgegenwärtige sowjetische Siegespathos zu hinterfragen: Statt auf den Sieg konzentriert sie sich auf die Kleinigkeiten des Kriegslebens und will vor allem die Perspektive der Kriegsteilnehmerinnen zeigen, deren Art zu erzählen von den Männern nicht geschätzt werde. Mit Alexijewitsch sprachen frühere Telefonistinnen, Sanitätsinstrukteurinnen, Panzersoldatinnen, Zugführerinnen und Scharfschützinnen. 2004 brachte Alexijewitsch eine überarbeitete und ergänzte Version heraus, in der sie von den Sowjetzensoren beanstandetes Material wieder aufnahm und auch die Argumente nennt, mit welchen diese Abschnitte von den Zensoren abgelehnt worden waren. Dass Frauen über den an der Front schwierigen Umgang mit ihrer Monatsblutung sprachen, war unzulässig. Dadurch werde die Frau erniedrigt, sie sei aber heilig. Erzählungen über Kannibalismus seien reine Lüge. Auch Berichte über wenig heldenhaftes Verhalten waren nicht willkommen, der Zensor deklarierte, Heroismus sei es, was das Land brauche, nicht Alexijewitschs Wahrheit.

Doch genau dieser Wahrheit widmete Alexijewitsch sich weiterhin. Auch bei »Die letzten Zeugen« (Poslednie swideteli; 1985, überarbeitet 2008, dt. 2014) wird deutlich, dass man bei allen Büchern Alexijewitschs darauf achten muss, wann sie herausgegeben bzw. nach welcher Vorlage sie übersetzt wurden. In der 2014 erschienen deutschen Übersetzung »Die letzten Zeugen" sind beispielsweise 101 Zeugenberichte enthalten, in einer bereits von der Autorin überarbeiteten russischen Ausgabe von 2004 waren 100 vertreten. Dafür fehlt in der deutschen Ausgabe das Vorwort der Autorin. Darin beschreibt sie, dass bei ihren Interviews für ihr erstes Buch über die Erfahrungen von Frauen im Krieg deren Kinder häufig dabeisaßen und zum ersten Mal hörten, was ihre Mütter erlebt hatten. Sie steuerten erinnerte Details bei. Das habe Alexijewitsch den Anstoß gegeben, Jahre später diejenigen aufzusuchen, die den Beginn des Großen Vaterländischen Kriegs 1941 als Kinder zwischen drei und 14 Jahren erlebten. Sie sei erschüttert gewesen von ihrer "schutzlosen Sprache«. Nur in diesem Vorwort findet sich auch der Verweis, dass Alexijewitsch sich auf Menschen in Belarus konzentrierte. Zwischen 1980 und 1985 führte Alexijewitsch ihre Gespräche mit Soja Masharowa (bei Kriegsbeginn 12 Jahre alt, »heute Postangestellte«), Wassja Sigaljow-Knjasew (6 Jahre, "heute Sporttrainer») und vielen anderen namentlich Genannten, die sich an den Krieg vor allem als an die Zeit erinnern, in der sie den Vater und / oder die Mutter verloren, für einige Jahre oder für immer. Erschießungen, brennende Häuser, Flucht, Kinderheim, Partisanen diese und viele weitere Details werden erzählt. Mit acht der ursprünglich um die 100 Zeuginnen und Zeugen Alexijewitschs wurden später Videointerviews geführt, die 2009 im russischen Fernsehen (Erster Kanal) ausgestrahlt wurden. Alexijewitsch war auch selbst an diesem Filmprojekt beteiligt. Sie hat zu zahlreichen Dokumentarfilmen Drehbücher verfasst und ist auch die Autorin von Theaterstücken. 
Der US-amerikanische Schriftsteller Larry Heinemann vergleicht die Berichte im nächsten Buchprojekt von Alexijewitsch "Zinkjungen " (Zinkowye maltschiki; 1989, dt. 1992) mit den ersten Erzählungen amerikanischer Vietnamrückkehrer: "this is what I saw, this is what I did, this is what I became.« Der Titel bezieht sich auf die Zinksärge, in denen die während der sowjetischen Intervention (1979-1989) in Afghanistan Getöteten an ihre Familien zurückgeschickt wurden. Die Särge waren verschweißt und durften nicht geöffnet werden. Dadurch wurde der Zustand der Leichen vor den Angehörigen verborgen.

Während sich Alexijewitsch bei der Wiedergabe der individuellen Erzählungen und Erinnerungen zurücknimmt, ist sie in Vor- und Nachwort und in Zwischenrufen präsent. Sie wolle die Wirklichkeit zeigen, denn nichts sei fantastischer - im Sinne von unglaublicher und extremer. Da sie nicht den Krieg, sondern die Gefühle darstellen will, die der Krieg auslöst, werden die Berichte der von ihr befragten Zeitzeugen oft genug zu Beichten. Die Absolution erteilt Alexijewitsch ihnen nicht, ebenso wenig kann sie den Taten und dem Leiden Sinn geben. Die Frage, wofür ein Soldat beide Beine und sein Freund sein Leben verloren hat, bleibt unbeantwortet.

Weil Alexijewitsch Teile des Buches vorab in Zeitschriften veröffentlichen ließ, konnte sie bei der Buchveröffentlichung am Ende auch Leserbriefe und Zusammenfassungen von Telefongesprächen berücksichtigen. Manche Beteiligten und vor allem die Mütter Gefallener sahen sich bzw. ihre Söhne nach wie vor als Helden, für sie war Alexijewitsch die Nestbeschmutzerin, die ihnen den Halt raubt. Eine Person wirft die Frage der Selbsterkenntnis auf: Vielleicht sei es gar nicht gut, soviel über sich selbst zu erfahren.

Heinemanns Vergleich mit dem Vietnamkrieg benennt den allgemeingültigen Aspekt von Alexijewitschs Arbeit. Sie befasst sich mit Themen, die einerseits speziell die Situation in der Sowjetunion und ihren Nachfolgestaaten betreffen, deren allgemein menschliche und überzeitliche Komponente - Leben und Sterben, Krieg und Töten - aber Menschen in aller Welt ansprechen und von ihnen verstanden werden können.

In "Im Banne des Todes« (russisch: Satscharowannye smertju, 1994, belarus. 1993, dt. 1994; Taschenbuch 1999 unter dem Titel „Seht mal, wie ihr lebt«) lässt Alexijewitsch die Verlierer des Zusammenbruchs der Sowjetunion zu Wort kommen. 17 Geschichten werden erzählt von Menschen, die einen Selbstmordversuch unternommen haben, oder den Hinterbliebenen von Selbstmördern: Familie, Nachbarn, Freunde. Als "literarisches Experiment «bezeichnet Nikolaj Kulaschenko diese Studie, und ihm zerreiße es das Herz, wie er, der Veteran des
Großen Vaterländischen Kriegs, nun beschimpft werde. Er sei "ein Zeuge, den niemand braucht«. Für Kulaschenko bringt die neue Freiheit nichts, und auch Pawel Stukalski, über den sein Freund Oleg Baschko berichtet, fand sich in der neuen Welt nicht zurecht. Aber während der 70jährige Kulaschenko beklagt, dass die Veteranen des Großen Vaterländischen Kriegs nicht mehr in die Schulen eingeladen werden, schimpft der 27jährige ehemalige Afghanistankämpfer Baschko, „Daß all die Kriegsveteranen, die uns früher in den Schulen besucht und mit ihren Medaillen angegeben haben, uns nicht die Wahrheit gesagt haben..." Alexijewitsch lässt die Aussagen in ihrer Widersprüchlichkeit aufeinanderprallen und schreibt auch mit, wenn es weh tut, wenn etwa Baschko seinem Freund hinterhertrauert: „Er war mein bester Freund. Wir haben zusammen Afghanistan und Karabach durchgemacht... Wir haben fremde Frauen vergewaltigt, wir haben getötet... Aber das ist der Krieg! Unsere Männerwelt!« Bei Natalja Paschkewitsch ist der Widerspruch gleich in der eigenen Biographie enthalten. Ihr Mann war im von Nazideutschland okkupierten Teil der Sowjetunion aufgewachsen und durfte deswegen später nicht studieren, auch eine Arbeitsstelle fand er kaum. Natalja Paschkewitsch klagt ihr Land - die Sowjetunion - und sich selbst an:

"Noch jahrelang wurde gegen das eigene Volk gekämpft, gegen die, die aus der Gefangenschaft kamen, die zur Zwangsarbeit nach Deutschland verschleppt waren, die nicht im Konzentrationslager umgekommen waren, nicht in Krematorien verbrannt waren... Mein Gott! Und ich habe marxistisch-leninistische Philosophie an der Hochschule unterrichtet! ... Die Partei ist das Gewissen, ist die Ehre... Und ich hatte überhaupt keine Zweifel..."

Alexijewitsch ergreift nicht das Wort. An einigen Stellen wird deutlich, dass die Sprecherin oder der Sprecher mit jemandem geredet und auf Fragen geantwortet hat. Alexijewitschs Gesprächspart taucht im Text aber nicht auf. Ihr Verdienst ist es, die Menschen zum Reden gebracht zu haben.

Dem Atomunfall in Tschernobyl widmete sie sich in »Tschernobyl - eine Chronik der Zukunft« (Tschernobylskaja molitwa, 1997; dt. 1997). Für dieses Buch wurde sie 1998 mit dem Leipziger Buchpreis für Europäische Verständigung und dem Sonderpreis "Das politische Buch« der Arbeitsgemeinschaft der Verleger, Buchhändler und Bibliothekare in der Friedrich-EbertStiftung ausgezeichnet. Auf einen kurzen Abriss der Fakten aus Sachbüchern wie der Weißrussischen Enzyklopädie lässt Alexijewitsch die teils anklagenden, teils abwiegelnden Erzählungen der Zeuginnen und Zeugen der Katastrophe folgen. Das Ereignis selbst interessiere 
Alexijewitsch nicht, wie sie im Abschnitt "Interview der Autorin mit sich selbst über versäumte Geschichte« zugibt, sondern es sind wieder die Einzelschicksale wie das der damals schwangeren Ljudmila Ignatenko. Ihr Mann Wasja war Feuerwehrmann und wurde bei dem Unglück rettungslos verstrahlt. In einem Krankenhaus in Moskau versorgte Ljudmila ihren Mann und beobachtete sein zwei Wochen dauerndes Sterben: „Die Farbe des Körpers... Blau... Rot... Graubraun...« Ljudmila verlor erst ihren Mann und später die Tochter, die vier Stunden nach der Geburt starb. Rückkehrerinnen wie Anna Petrowna Badajewa dagegen leugnen die Folgen des Unfalls: "Ich denke, daß es gar kein Tschernobyl gibt, das haben sie sich nur ausgedacht...« Damals nach Tschernobyl zum Aufräumen abkommandierte Soldaten berichten, sie hätten erst Jahre später begonnen nachzudenken. Damals waren sie tagsüber im Sperrgebiet und verfolgten abends im Fernsehen die Fußballweltmeisterschaft. Einer von ihnen verbrannte nach dem Einsatz alle Kleidungsstücke bis auf sein Käppi, das er seinem kleinen Sohn schenkte. Dieser bekam später einen Hirntumor.

Mit "Secondhand-Zeit" (Wremja second-hand, 2013, dt. 2013) wurde Swetlana Alexijewitsch endgültig auch im westlichen Ausland als Erforscherin des sowjetischen und postsowjetischen Lebens bekannt. In dieser umfangreichen Alltagsstudie ist ihre Stimme intensiver zu vernehmen als in den vorangegangenen Publikationen. Nicht umsonst betitelt sie das Vorwort mit "Aufzeichnungen einer Beteiligten«. Sie dokumentiert ihre Interaktion mit 20 Zeitzeuginnen und Zeitzeugen sowie ihre Eindrücke waus Straßenlärm und Küchengesprächen" der Jahre 1991-2001 bzw. 2002 2012. Einige Erzählungen sind schon aus "Im Banne des Todes« bekannt. Zum neuen Material gehören Alexijewitschs persönliche Eindrücke und Notizen, etwa von einer Parade zum ersten Mai in den 1990er Jahren, bei der die einen auf die "Amerika-Lakaien" schimpften, während die anderen »Beerdigt Lenin« riefen. Oder wie sie den desillusionierten Trinkern zuhört, die einander ins Wort fallen. Der eine preist den Sozialismus, der den Menschen gezwungen habe »in der Geschichte zu leben... an etwas Großem teilzuhaben«, wogegen der nächste protestiert: "Scheiße! Wir sind ja so spirituell, wir sind ja so besonders!« Aus der Gesprächspartnerin Margarita Pogrebizkaja, Ärztin, 57 Jahre alt, bricht es regelrecht heraus: „Vielleicht war das ja ein Gefängnis, aber mir war in diesem Gefängnis wärmer.»

\section{Preise}

Für ihr schriftstellerisches Schaffen wurde Alexijewitsch mit zahlreichen Preisen ausgezeichnet, darunter dem Lenin-Komsomol-Preis 1986 (für "Der Krieg hat kein weibliches Gesicht«), dem Leipziger Buchpreis zur Europäischen Verständigung (Hauptpreis) 1998, dem ErichMaria-Remarque-Friedenspreis 2001 und dem Friedenspreis des Deutschen Buchhandels 2013.

Alexijewitsch ist weiterhin als Journalistin tätig und veröffentlicht im In- und Ausland Artikel zu politischen Themen. So erschien im April 2014 unter dem Titel »Wer nicht jubelt, ist ein Volksfeind « ein Beitrag zu Russlands Annexion der Krim in der Frankfurter Allgemeinen Zeitung, in dem Alexijewitsch die Situation in Russland mit den Worten beschreibt: "Wir haben nicht die Krim wiederbekommen, sondern die Sowjetunion.«In dieses russische "wir« schließt Alexijewitsch sich ein und verweist damit auf ihre eigene (post-)sowjetische Sozialisation. Mit dem heutigen Russland identifiziert sie sich dabei ebenso wenig wie mit dem heutigen Belarus. Und doch ist Belarus durch den Nobelpreis international sichtbarer geworden.

Nur drei Tage nach der Bekanntgabe, dass der Literaturnobelpreis 2015 an Swetlana Alexijewitsch geht, erreichte Lukaschenka in den belarussischen Präsidentschaftswahlen wieder einmal ein Rekordergebnis (83,5\%). Die Hauptstadt Minsk taucht dieser Tage meist in Zusammenhang mit den Vermittlungsgesprächen im Ukrainekonflikt auf, bei denen Lukaschenka der Gastgeber ist. Doch auch wenn der belarussische Dissident Andrej Sannikow die Nobelpreisvergabe an Alexijewitsch als Zeichen von außen deutet, dass man Belarus nicht vergessen habe, und sich freut, dass Belarus endlich Anerkennung als Literaturland finde, widersetzt sich Alexijewitschs Schaffen nationaler Vereinnahmung.

\section{Über die Autorin}

Dr. Yvonne Pörzgen ist Geschäftsführerin des BA Integrierte Europastudien an der Universität Bremen. Sie forscht zur russischen und polnischen Literatur v. a. des 19. bis 21. Jahrhunderts. In ihrem Habilitationsvorhaben befasst sie sich mit dem freien Willen in slavischen Literaturen.

\section{Lesetipps:}

- Swetlana Alexijewitsch: Secondhand-Zeit. Leben auf den Trümmern des Sozialismus. Aus dem Russischen von Ganna-Maria Braungardt. München: Hanser Berlin, 2013.

- Swetlana Alexijewitsch: Die letzten Zeugen: Kinder im Zweiten Weltkrieg. Aus dem Russischen von Ganna-Maria Braungardt. Überarbeitete, aktualisierte Neuausgabe. München: Hanser Berlin, 2014. 


\title{
Belarussische Dilemmata. Die Rezeption des Nobelpreises für Swetlana Alexijewitsch in Belarus
}

\author{
Von Felix Ackermann, Vilnius
}

\section{Einleitung}

Die Reaktionen auf die offizielle Verkündung des Nobelpreises für Literatur 2015 an Swetlana Alexijewitsch in Belarus waren durchwachsen: Viele belarussische Intellektuelle kommentierten, es sei ein großer Tag für die belarussische Literatur. Dennoch war in fast allen Gratulationen ein Aber zu lesen. Swetlana Alexijewitsch wurde als Chronistin ausgezeichnet, die denjenigen eine Stimme gibt, die sonst im Westen und Norden des Kontinents, aber auch im eigenen Land nicht erhört werden. Dennoch ist das Verhältnis vieler Menschen in Minsk zu ihrer Nobelpreisträgerin gespalten. Ein wichtiger Grund ist, dass mit Swetlana Alexijewitsch eine Autorin den Preis erhält, die in ihrem Werk bewusst die Grenzen zwischen Journalismus, Oral History und dem Weben einer Metaerzählung auflöst. Ihre über fünf Bücher entwickelte Methode die Stimmen ihrer Protagonisten zu verdichten und in eine Geschichte der sowjetischen Gesellschaft und ihres Zerfalls zu fügen, erscheint einigen in Minsk zu sowjetisch. Andere finden sie nicht belarussisch genug.

Der Widerspruch zwischen der prinzipiellen Anerkennung des Werkes von Swetlana Alexijewitsch und der konsequenten Selbstleugnung der belarussischen kulturellen Elite ist charakteristisch für ein Land, in dem die Nationsbildung im gesamten 20. Jahrhundert keinen eindeutigen und umfassenden Prozess der kulturellen und politischen Mobilisierung entlang nationaler Linien hervorgebracht hat. Die Republik Belarus ist heute dennoch als Nationalstaat organisiert. Und auch der Nobelpreis selbst wird zwar an Individuen vergeben, aber diese haben in der medialen Wahrnehmung stets eine Nationalität, an die eine gewisse Erwartung geknüpft ist: Die Nobelpreisträgerin erhält durch die Auszeichnung die Legitimität vor einer Weltöffentlichkeit über ihr Land zu sprechen, ja für ihr Land zu sprechen. Da Swetlana Alexijewitsch aber den nationalen Charakter ihres Landes weitgehend leugnet und sich und ihre Bücher stattdessen in einem breiteren post-sowjetischen, russischsprachigen Kontext verortet, sind die Reaktionen auf ihren Preis in Minsk vor allem charakteristisch für belarussische Dilemmata.

\section{Eine Biographie des kollektiven Verlusts}

Auf dem Cover der 2013 erschienen deutschen Fassung ihres Buches "Secondhand-Zeit« ist eine ältere Frau zu sehen, die mit Begeisterung eine sowjetische Flagge über den Minsker Oktoberplatz trägt. In "Secondhand-Zeit" verfasste die belarussische Autorin die kollektive Biogra- phie derjenigen, die mit der sowjetischen Utopie aufgewachsen waren und deren Lebenswege noch heute von ihrem plötzlichen Untergang geprägt sind. Das Buch skizziert die Euphorie der Perestroika und ihren Höhepunkt, die Verteidigung des Moskauer Weißen Hauses gegen den Putsch im August 1991, ebenso wie die folgende Spaltung der post-sowjetischen Gesellschaft in diejenigen, die die Sowjetunion um jeden Preis und diejenigen, die sie um keinen Preis zurückhaben wollten. Sie zeigt anhand einzelner Biographien, wie der Zusammenbruch das Lebenswerk Einzelner zerstörte, aber auch den Lebensstil und die Gewissheiten einer ganzen Gesellschaft grundlegend veränderte. Den wirtschaftlichen Niedergang der 1990er Jahre und die Marginalisierung der an den sowjetischen Küchentischen sozialisierten Intelligenzija beschreibt die Autorin besonders prägnant. In der Einleitung schreibt sie, dass der sowjetische Mensch keine Utopie gewesen sei, sondern über Generationen hinweg ganz real im sowjetischen Alltag existiert habe. Während sie in den folgenden Kapiteln stets ihre Interviewpartner zu Wort kommen lässt, erfährt der Leser am Anfang beiläufig, dass die Autorin sich selbst auch zum Kreise dieser sowjetischen Menschen zählt. Es handelt sich bei "Secondhand-Zeit« um eine Meditation des "Abschieds von der kommunistischen Zeit». Alexijewitsch konzipierte das Buch als individuelle und kollektive Therapie gegen den post-sowjetischen Phantomschmerz, der nicht mit Nostalgie zu verwechseln ist.

\section{Chronistin der sowjetischen Utopie und ihres Untergangs}

Swetlana Alexijewitsch schloss mit "Secondhand-Zeit" einen Zyklus von fünf Werken über den »roten Menschen" $a b$, mit dem sie sowjetische Kulturgeschichte schrieb. Nach dem Studium in Minsk arbeitete sie in den 1970er Jahren als Journalistin an verschiedenen Orten der Belarussischen Sozialistischen Sowjetrepublik. Ohne staatliche Unterstützung begann sie in der gesamten Sowjetunion Frauen zu interviewen, die als Soldatinnen am Zweiten Weltkrieg teilgenommen hatten. Die daraus entstandene literarisch-dokumentarische Collage »Der Krieg hat kein weibliches Gesicht « konnte erst 1985 als Gesamtausgabe erscheinen, weil die Autorin durch die Auswahl, Verdichtung und Montage von Zitaten zentralen Vorstellungen über den Zweiten Weltkrieg widersprach, der unter Leonid Breschnew als Großer Vaterländischer Krieg zum Fundament sowjetischer Identitätsstiftung geworden war. 
Ihr Spiel mit dem weiblichen Geschlecht des russischen Worts für Krieg - wojna - verwies implizit auch auf den männlich geprägten Kult eines Krieges, der vor allem Leid über die sowjetische Gesellschaft gebracht hatte. Die Glasnost-Zeit mit ihrer neuen Form kritischer Öffentlichkeit prägte sie mit diesem Band ebenso wie mit dem folgenden Werk "Die letzten Zeugen", in dem sie die individuellen und kollektiven Folgen von Gewalt in der sowjetischen Gesellschaft nachzeichnet. Bereits 1990 erschienen erste Texte über die sowjetische Intervention in Afghanistan in Moskauer Zeitschriften. Auch hier stellte sie das Leid der einzelnen Soldaten, ihrer Mütter und Geschwister durch ihre Montagetechnik in einen größeren Zusammenhang. 1992 erschienen diese Texte als "Zinksoldaten" erstmals in gebundener Form auf Russisch.

\section{Die künstlerische Herstellung kollektiver Authentizität}

Die einzelnen Interviewsequenzen verdichtet Swetlana Alexijewitsch durch eine starke Fokussierung auf einzelne Schicksale und deren gezielte Montage. Sie versieht diese nur mit wenigen zusätzlichen Informationen der Leser erfährt Alter, Beruf, seltener auch den Namen und den Ort. So verbleiben die als "authentisch" markierten Zitate oft ohne Informationen über den Kontext der Auswahl, über die Bedingungen der Interviews. Doch nach längeren Passagen, in denen die Autorin ihre Protagonistinnen und Protagonisten zitiert, beschreibt sie ihren eigenen Gedankenfluss oder die Situation, in der ein Interview entstanden ist. Noch in "SecondhandZeit" wird ihr gezieltes Ineinanderschieben unterschiedlicher Biographien durch die konsequente Verwendung der ersten Person Plural verstärkt. Alexijewitsch notiert zwar das Leid des Einzelnen, doch verwendet sie dieses allein als Versatzstück eines gedachten Kollektivs des sowjetischen Volkes, das seine Heimat verloren hat.

In "Zinksoldaten« beschreibt die Autorin ihre eigene Methode: „Wieder mein Weg - von Mensch zu Mensch, von Dokument zu Dokument. Jede Beichte - wie ein Portrait in der Malerei: Niemand würde "Dokument« dazu sagen, man sagt »Bild«. Man spricht vom Phantasiegehalt der Realität." [1] Demnach interessierte sich die Autorin für die Gedanken, Wünsche und Ängste des einzelnen Menschen. Durch den starken Auswahl- und Montageprozess verstärkt sie die Stimmen ihrer Interviewpartner. Selbst verstummte Stimmen der »sowjetischen Zivilisation« werden durch ihre Technik zu einer Quelle. Besonders stark wirkt dies im Falle ihrer intensiven Recherchen zu (post)sowjetischen Selbstmördern wie Timerian Zinatow, einem Verteidiger der Festung Brest, der im Alter von 77 Jahren dorthin zurückkehrte, um sich das Leben zu nehmen. [2] Dieser Prozess der Überführung eines Interviews in einen als künstlerisch markierten Text ist verbun- den mit einer starken symbolischen Aufladung, die auch mit dem Mittel des Pathos arbeitet. Dieser Effekt wird verstärkt durch den gezielten Einsatz von Zitaten russischer und sowjetischer Literaturklassiker, die in der Regel als solche markiert sind oder vom kanonisch geschulten (post-)sowjetischen Leser aber auch ohne Verweis auf die Quelle erkannt und gedeutet werden können.

Diese Vermengung und gleichzeitige Verdichtung von "authentischen" Quellen ist das, was Alexijewitsch als Autorin ausmacht. Ihre Bücher sind gut zu lesen. Die Sätze kleben nicht aneinander wie in historiographischen Schriften, die Oral History auf der Metaebene analysieren. Und ihre grundlegende Frage nach dem »Schicksal» derjenigen, die noch bis vor kurzem das sowjetische Volk ausgemacht haben, formuliert ein starkes Erkenntnisinteresse, dass es ihr ermöglicht, mit jedem einzelnen Buch auf den Grund dieser Gesellschaft zu gehen. Schicksal in Anführungszeichen, weil auch Swetlana Alexijewitsch Grundannahmen über das Leben des Menschen und die Schicksalshaftigkeit dieses Menschen mit ihren Lesern und vielen Historikern in ihrem Land teilt.

Iryna Kaschtaljan, die Gründungsdirektorin des Belarussischen Oral History Archivs in Minsk ist begeistert von Alexijewitsch und dem Nobelpreis, weil sie den Preis auch als Anerkennung ihrer eigenen Arbeit und des Konzepts vom »kleinen Menschen" sieht, der dem Lauf der »großen Geschichte " weitgehend ausgeliefert ist. In ihrer Dissertation beschrieb Kaschtaljan die Leiden der Nachkriegsgesellschaft im Westen von Belarus auf einer breiten Grundlage von mündlichen Quellen. In Minsk wurde diese Arbeit von der obersten Kontrollinstanz der Akademie der Wissenschaften nicht zugelassen - nicht nur weil Kaschtaljan Positionen vertritt, die mit der offiziellen Staatsideologie nicht vereinbar sind. Die Verlässlichkeit mündlicher Quellen gilt in belarussischen staatlichen Institutionen noch immer als zu gering. Und hierin sieht die junge Historikerin den Wert von Alexijewitschs Büchern. Nun sei für jeden in Belarus erkennbar, dass das gesprochene Wort Grundlage für eine historische Erzählung sein kann.

Durch ihre betont dokumentarisch-künstlerische Arbeitsweise gelingt Alexijewitsch etwas, was Historikern - in Belarus wie im Westen Europas - nur selten gelingt: Sie schafft durch Verdichtung einen Text, den man als Leser wie ein Epochengemälde auf sich wirken lassen kann. Dass die Texte nicht als historische Quellen codiert sind, liegt auf der Hand. Der Hauptunterschied zu einer strikt historiographischen Arbeitsweise, die stark mit Dokumenten und Zitaten arbeitet, liegt darin, dass die Autorin ihre eigene Sicht oft nur indirekt über die Auswahl der Zitate und ihr Arrangement sichtbar macht. Der Leser sieht Alexijewitsch als Fragende nur implizit, sie tritt wie bei den frühen Video-Interviews der ersten Phase der klassischen Holocaust-Oral-History nicht als Akteu- 
rin in Erscheinung, obwohl sie gleichzeitig durch die von ihren Fragen strukturierte Narration omnipräsent ist.

Genau hier liegen die Grenzen der Verwendbarkeit ihrer Texte für die Geschichtswissenschaft - im Westen wie in Belarus und Russland gleichermaßen. Der kritische Leser erfährt in den Büchern nur wenig über die Entstehungsbedingungen der Texte. Sie sind geschützt vom Nimbus des Kunstwerks und leben doch vom Anspruch auf "Authentizität« der mündlichen Quellen. Als literarische Autorin genießt Swetlana Alexijewitsch bei ihren Lesern das Urvertrauen der Leser in ihre Autorin. Als Erzählerin schützt sie genau das Konstrukt der künstlerischen "Authentizität« vor der konsequenten Offenlegung ihrer Arbeitsweise. Diese Einschränkung gilt allerdings auch für viele Historiker in Belarus und jenseits davon. Obwohl sie zwar auf eine Reihe von zitierten Quellen - ob schriftlich oder mündlich - verweisen, ist für den Leser nicht erkenntlich, nach welchen Kriterien sie ausgewählt und nach welcher Methode sie miteinander in Bezug gesetzt wurden. Als Autorin von Belletristik genießt Alexijewitsch jedenfalls volle Immunität gegen diesen Vorwurf. Dennoch werden die in ihren Werken zitieren Protagonisten von Historikern im Westen wie im Osten als Quellen zitiert ohne die Lücke zwischen dieser Immunität und der Historizität und der Narration selbst zu thematisieren.

\section{Die Rezeption in Belarus, Russland und Deutschland}

In Belarus, wo Swetlana Alexijewitsch unter sowjetischer Herrschaft in einer belarussisch-ukrainischen Familie aufwuchs, wurden ihre Werke seit Ende der 1990er Jahre nicht mehr gedruckt. Staatliche Stellen können bis heute mit ihrer Dokumentarprosa ebenso wenig anfangen wie die national argumentierenden Akteure der Opposition. Während die einen das Erbe der Sowjetunion beschmutzt sehen, ist den anderen Alexijewitschs Werk nicht stark genug auf Belarus fokussiert.

Die Autorin schreibt aber, wie in "SecondhandZeit» durch das konsequent verwendete »Wir« klar wird, bewusst nicht für einen national definierten Leser etwa in Minsk oder Kiew, sondern gezielt für jenen einstigen sowjetischen Menschen, dessen Sprache Russisch ist, der Dostojewski, Tolstoi, aber auch Solschenizyn und Schalamow gelesen hat. Ihre in Moskau verlegten russischsprachigen Originalwerke werden in der gesamten ehemaligen Sowjetunion und durch diverse Emigrationswellen auch im westlichen Europa und Nordamerika im Original gelesen. Sie wurden in über 30 Sprachen übersetzt.

In Deutschland und Frankreich wurde die Autorin nach der Tschernobyl-Katastrophe besonders stark wahrgenommen, weil sie in ihrem Buch »Tschernobyl. Chronik der Zukunft« eine neue Dimension menschlichen Lebens und Sterbens nach der Reaktorkatastrophe beschrieb.
Bereits 2013 erhielt sie nach vielen anderen internationalen Ehrungen den Friedenspreis des Deutschen Buchhandels. Die Jury begründete die Entscheidung damals damit, dass Swetlana Alexijewitsch »die Lebenswelten ihrer Mitmenschen aus Weißrussland, Russland und der Ukraine nachzeichnet und in Demut und Großzügigkeit deren Leid und deren Leidenschaften Ausdruck verleiht." [3]

Weitgehend ausgeblendet wird bei der Rezeption von Swetlana Alexijewitsch, dass sie über zehn Jahre selbst als Emigrantin mit Stipendien in Deutschland, Schweden und Frankreich gelebt hat und sich dadurch selbst von ihrem Gegenstand und ihren Interviewpartnern temporär, räumlich und sozial entfernt hat. Dennoch kehrte sie für die Interviews immer wieder zurück - in die post-sowjetische Vergangenheit. Dass sich in der Gegenwart Russlands, aber auch in Belarus neue kulturelle Formen entwickelten, die jenseits der Logik der sowjetischen Utopie und ihres Endes funktionieren, ist für das Projekt der Autorin nicht relevant. Sie hatte nach der Verleihung des Friedenspreises 2013 unter der belarussischsprachigen, neuen kulturellen Elite in Belarus für einen Skandal gesorgt, indem sie in einem Interview mit der Frankfurter Allgemeinen Zeitung einem alten sowjetischen Argumentationsmuster über die Kulturen der nichtrussischen »Titularnationen" in der Sowjetunion folgte und behauptete, Belarussisch würde sich nicht als Literatursprache eignen, weil es eben doch eine Bauernsprache sei. Das war eine öffentliche Schelte für diejenigen in Minsk, die seit einiger Zeit munter, frech und mit einigem Erfolg auf Belarussisch schreiben.

Doch Swetlana Alexijewitsch kehrte 2011 offiziell nach Minsk zurück und lebt dort seither wieder gegenüber dem Denkmal für die belarussischen Opfer des sowjetisch-afghanischen Kriegs, das im Volksmund "Insel der Tränen" genannt wird. Es gibt unter Afghanistan-Veteranen eine Legende, dass das Denkmal, das offiziell »Insel des Heldentums und Trauer« heißt, mit voller Absicht vor Swetlana Alexijewitschs Wohnblock errichtet wurde, denn organisierte Veteranen sahen ihre soldatische Ehre durch das Buch »Zinkjungen« verunglimpft.

\section{Minsk als Teil der Russischen Welt}

Bereits zwei Jahre vor der Bekanntgabe des Nobelpreises war "Secondhand-Zeit« im Minsker Lohwinau Verlag in belarussischer Übersetzung erschienen. Damit war ein zentrales Argument von Alexijewitschs Künstlercredo als in ihrer Heimat dauerhaft Verfolgter überkommen. Es ist doch möglich ihre Bücher in Minsk zu drucken. Doch für die Finanzierung ihres Lebensunterhalts und den Verkauf der Bücher der Autorin im Westen war stets förderlich, dass ihre Bücher in Minsk seit Ende der 1990er Jahre nicht erscheinen konnten. Da Alexijewitsch aber auf Russisch schreibt und Belarus ein kleiner Teil des russischsprachigen Buchmarktes ist, waren ihre Bücher nie aus Minsk 
verschwunden. Alle Neuigkeiten der kommerziellen Moskauer Verlagswelt sind hier stets druckfrisch erhältlich. Inklusive der Bücher von Swetlana Alexijewitsch.

Noch zum Beginn der 1990er Jahre wurden im Zuge der "Adradschenie" genannten nationalen RenaissanceBewegung alle damals erschienen Werke von Alexijewitsch ins Belarussische übertragen und hatten auch in Minsk große Auflagen. Einzelne Texte fanden sogar Einzug in die Lehrpläne. Doch als die Autorin Ende der 1990er Jahre den zunehmend autoritären Führungsstil des Präsidenten Alexander Lukaschenka weiterhin öffentlich kritisierte, veränderte sich die Lage. Fünfzehn Jahre lang traute sich kein Minsker Verlag mehr, ihre Bücher in Belarus zu drucken. Alexijewitsch hatte zunehmend Probleme öffentlich aufzutreten und musste das Land verlassen, um sich mit westlicher Unterstützung ihren Lebensunterhalt zu sichern.

Dennoch waren ihre Bücher in Belarus stets erhältlich. Da die Autorin auf Russisch schreibt, ist es Konsequenz des russischsprachigen Markts, dass ihre Bücher in Moskau verlegt werden und damit in Minsk weiterhin in den Buchhandlungen zu finden waren und sind. Und in jeder Minsker Buchhandlung kann man auch belarussischsprachige Werke kaufen: Die Produktion der staatlichen Verlage, die jedes Jahr neue Bildbände von Minsk und den Bezirksstädten des Landes verlegen, weil ihnen nichts einfällt, was sonst jemand auf Belarussisch kaufen würde. In einigen großen Buchläden gibt es auch eine Ecke für die alternative Produktion von Historikern wie Iryna Kaschtaljan und eine noch kleinere mit den Bänden der Schriftsteller, die aus Prinzip auf Belarussisch schreiben und die ihren eigenen unabhängigen PEN-Club haben.

Die Übermacht des Russischen und die geringe Bedeutung von Belarussisch im Alltag der modernen belarussischen Nation werden im Internet noch deutlicher. Auf Wikipedia fallen weniger als 2 Prozent aller Serveranfragen aus Belarus auf eine der beiden belarussischsprachigen Versionen des Online-Lexikons. Das erklärt, warum Swetlana Alexijewitsch so belarussisch wie ihre ganze Gesellschaft ist, obwohl sie Belarussisch öffentlich den Rang als Literatursprache abspricht. Es erklärt aber auch, warum viele Schriftsteller, Historiker und Philologen in Minsk ein gespaltenes Verhältnis zu Swetlana Alexijewitschs Werk und nun auch zu ihrem Nobelpreis haben.

\section{Kultur und Politik}

Diese Analyse des Minsker Buchmarktes unter ökonomischen Gesichtspunkten stellt nicht in Abrede, dass Belarus heute ein autoritäres Regime ist, in der das Wort eine besondere Bedeutung hat. Der Lohwinau-Verlag sah sich kurze Zeit nach dem Erscheinen der belarussischen Version von "Secondhand-Zeit" auf Belarussisch 2014 staatsanwaltlichen Ermittlungen wegen angeblicher krimineller Machenschaften ausgesetzt. Belarussische Politologen deu- teten das als klares Zeichen einer Warnung durch Alexander Lukaschenka im Vorfeld der Präsidentschaftswahlen. Damit ist die Geschichte von der politischen Verfolgung in Belarus doch wieder stimmig: Selbst die belarussische Ausgabe von Alexijewitsch stand im Zuge des Verfahrens gegen den Verlag kurz vor dem staatlichen Einstampfen.

Und das war kein Zufall, denn der Buchladen des Verlagshauses Lohwinau ist der einzige in Minsk, der dem Moskauer Massenmarkt erfolgreich eine zeitgenössische, eigene Version von Kultur entgegenstellt. Die Sprache dieser Gegenwart ist Belarussisch, aber es gibt hier Regale mit den wichtigsten Neuerscheinungen auf Russisch, auf Polnisch, auf Englisch und auf Deutsch. Dazu passt bestens, dass ihr belarussischer Verleger Lohwinau selbst nur mit einem Augenzwinkern Belarussisch spricht. Das muss er auch nicht in einer russischsprachigen Stadt. Und das schmälert auch seine Verdienste für die belarussische Kultur in keiner Weise. Dass der Verlag nach der Verkündung des Nobelpreises zu den ersten offiziellen Gratulanten in Belarus ohne wenn und aber wurde, war selbstverständlich.

Alle belarussischen Dilemmata gelten im Moment des Triumphs als Belanglosigkeiten. Aus Minsker Sicht wurde mit dem Nobelpreis nicht nur das Werk der Autorin, sondern indirekt auch die belarussische Nation geadelt. Doch da Belarus ja immer noch eine Diktatur ist, wird der daraus resultierende Rummel nicht von den großen staatlichen Zeitungen des Landes befeuert. Sie folgen der Präsidialadministration und vermerken die höflichen Glückwünsche von Alexander Lukaschenka. Der zentrale Ort für die weitgehend positiven Reaktionen auf die Stockholmer Entscheidung ist Facebook. Viele, die Alexijewitsch entweder wegen mangelndem Patriotismus oder wegen ihres öffentlichen Auftretens als Grand Dame der belarussischen Dissidenz kritisiert hatten, überwanden ihre inneren Widerstände und stimmten nun in halboffiziellen Kommuniques an ihrer Wandzeitung in dem sozialen Netzwerk aus Amerika in den öffentlichen Freudentaumel ein. Hunderte Likes waren der Dank der Facebook-Freunde etwa des Minsker Kunsthistorikers Siarhej Chareuski, der vorher nicht als bekennender Alexijewitsch-Fan auffällig geworden war.

Doch viele gratulierten am Ende wohl eher sich selbst und der belarussischen Nation statt der Autorin aufrichtig für ihr Werk zu danken. So blieben viele semioffiziellen Gratulationsnachrichten der westlich gesonnenen Zeitgenossen auf Facebook mit einem nicht klar benannten, aber spürbaren Aber versehen. Genau deshalb sah sich der unter jungen Leuten gefeierte Schriftsteller Wiktor Martinowitsch, dessen belarussischsprachigen Werke auch ins Deutsche übertragen wurden, zu einem Rundumschlag der Verteidigung genötigt: "Das ist ein Signal an die Belarussen: Landsleute, wir besitzen eine eigene Literatur! Und sie ist nicht schlechter als 
andere Weltliteraturen! Lest bitte die Bücher der Preisträgerin, wenn Ihr es noch nicht getan habt!«

Es ist von außen kaum verständlich, dass das Kleinklein dieser Minsker Reaktionen nur selten Bezug auf die außenpolitische Dimension des Stockholmer Komitees nahm. Dieses hatte nicht ohne Hintergedanken die Entscheidung drei Tage vor der noch längerfristiger geplanten Wiederwahl von Alexander Lukaschenka zum Präsidenten verkündet. Während die Kommentatoren in aller Welt genau dieses Signal erkannten und lobten, verpuffte es in Minsk, weil hier bereits bekannt war, wie die Wahl am Sonntag nach der Bekanntgabe ausge- hen würde: wie immer. Dennoch verlieh der Nobelpreis den kritischen Worten von Swetlana Alexijewitsch bei ihrer ersten Pressekonferenz neue Legitimation. Und für alle in Minsk war klar: Für wenige Tage werden Redakteure in aller Welt nach genau diesem Verhältnis zwischen Alexijewitsch und der Diktatur in Belarus fragen.

Nur drei Wochen nach der Bekanntgabe der Entscheidung des Stockholmer Komitees brach Lukaschenka den kurzen kulturellen Burgfrieden seiner fünften Amtszeit und griff bei einer Musikveranstaltung in der Minsk Arena indirekt die Schriftstellerin an. Damit ist in Minsk wieder alles beim Alten.

\section{Über den Autor}

Dr. Felix Ackermann ist DAAD Langzeitdozent für angewandte Kulturwissenschaften an der Europäischen Humanistischen Universität, einer belarussischen Exilhochschule in der litauischen Hauptstadt Wilna.

\section{Im Text zitierte Quellen}

[1] Swetlana Alexijewitsch, Zinkowye maltschiki, Moskva 2001, S. 13.

[2] Swetlana Alexijewitsch, Wremja second-hand, Moskwa 2013, Kindle-Edition, Position 4045.

[3] <http://www.boersenverein.de/445722/?aid=626370>, 10.10.2013.

\section{Die Bekanntheit von Swetlana Alexijewitsch in der russischen Bevölkerung}

\section{Grafik 1: Haben Sie von der belarussischen Schriftstellerin Swetlana Alexije- witsch gehört, die in diesem Jahr den Nobelpreis für Literatur er- halten hat?}

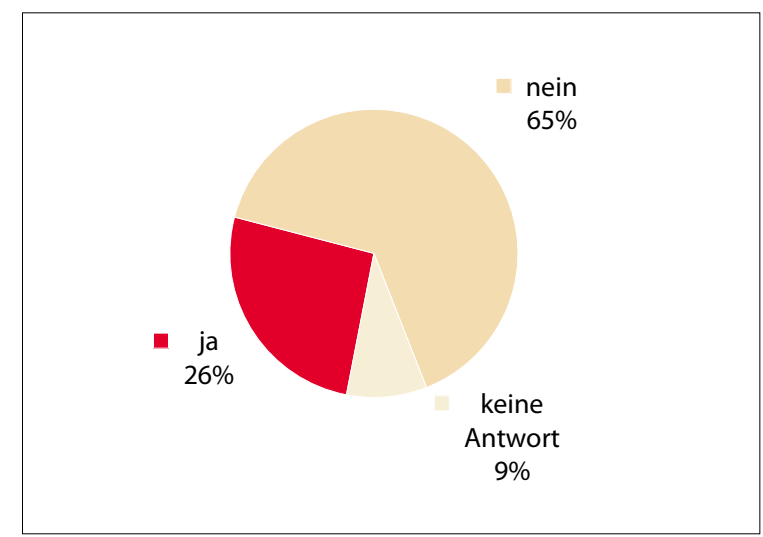

Grafik 2: Welche Bücher von Swetlana Alexijewitsch haben Sie gelesen? (Prozentanteil aller Befragten, mehrere Antworten möglich)

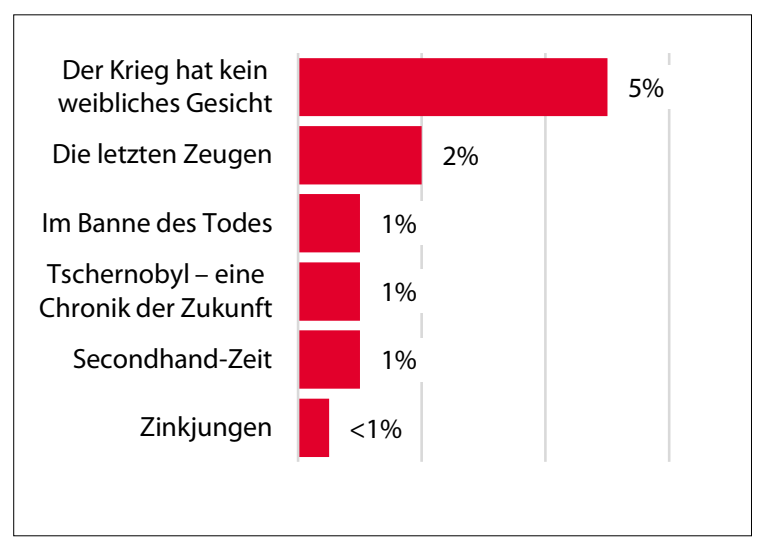

Quelle: Repräsentative Umfrage unter der Bevölkerung der Russischen Föderation, Lewada-Zentrum, Ende Oktober 2015, <http:// www.levada.ru/2015/10/30/uznavaemost-svetlany-aleksievich/> 


\section{Oktober - 29. November 2015}

\begin{tabular}{|c|c|}
\hline 12.10 .2015 & $\begin{array}{l}\text { Die Kampagne »Recht auf Wahl-2015«, die Vertreter acht belarussischer politischer Parteien und Organisa- } \\
\text { tionen vereinigt, erklärt, dass die von der Zentralen Wahlkommission bekanntgegebenen vorläufigen Wahl- } \\
\text { ergebnisse }(83,49 \% \text { der Stimmen für Lukschenka) nicht glaubwürdig sind. }\end{array}$ \\
\hline 12.10 .2015 & $\begin{array}{l}\text { Die Ergebnisse der Wahlbeobachtung durch die Kampagne »Menschenrechtsaktivisten für freie Wahlen« wer- } \\
\text { den in Minsk präsentiert. Menschenrechtsorganisationen melden zahlreiche Verletzungen sowohl der inter- } \\
\text { nationalen, als auch der belarussischen Wahlstandards während des Wahlprozesses. 92,3\% der Beobachtun- } \\
\text { gen lassen darauf schließen, dass die Stimmenauszählung nicht für alle Mitglieder der Wahlkommissionen } \\
\text { und Beobachter offen und transparent gewesen ist. }\end{array}$ \\
\hline 12.10 .2015 & $\begin{array}{l}\text { Kent Härstedt, Koordinator der kurzfristigen OSZE-Beobachtermission in Belarus und Vizevorsitzender der } \\
\text { PV OSZE, erklärt während einer Pressekonferenz zu den vorläufigen Ergebnissen der Wahlbeobachtung, dass } \\
\text { Belarus noch eine große Anstrengungen leisten müsse, um den Demokratie-Verpflichtungen der OSZE ent- } \\
\text { sprechen zu können, vor allem was die Stimmenauszählung betrifft. Das bestätige die Notwendigkeit für den } \\
\text { politischen Willen zu Systemreformen, so Härstedt. }\end{array}$ \\
\hline 12.10 .2015 & $\begin{array}{l}\text { Der Rat der EU einigt sich darauf, restriktive Maßnahmen (Einreiseverbote und Kontensperrungen) gegen } \\
\text { eine Reihe von Personen in Belarus, sowie einige Unternehmen für vier Monate - Ende Oktober } 2015 \text { bis } \\
\text { Ende Februar } 2016 \text { - auszusetzen. Der offizielle Beschluss soll Ende Oktober gefasst werden, so der Staatsse- } \\
\text { kretär für Europa des französischen Außenministeriums, Harlem Désir. }\end{array}$ \\
\hline 12.10 .2015 & $\begin{array}{l}\text { Die USA begrüßen den friedlichen Verlauf der Wahlen in Belarus, sind allerdings von ihren nicht-freien Cha- } \\
\text { rakter enttäuscht, erklärt der stellvertretende Pressesprecher des US-Außenministeriums Mark Toner. }\end{array}$ \\
\hline 12.10 .2015 & $\begin{array}{l}\text { Drei Fußballfans werden wegen »Hooliganismus« (angeblich wegen des Tragens von Schals mit dem Natio- } \\
\text { nalwappen »Pahonja«, das von den Behörden als oppositionell betrachtet wird) von einem Barysauer Gericht } \\
\text { jeweils zu einer Geldstrafe in Höhe von 1,8 Mio. BYR (umgerechnet knapp } 92 \text { Euro) verurteilt. }\end{array}$ \\
\hline 13.10 .2015 & $\begin{array}{l}\text { Die EU kündigt in einer gemeinsamen Erklärung der Hohen Vertreterin der EU für die Gemeinsame Außen- } \\
\text { und Sicherheitspolitik Federica Mogherini und des EU-Kommissars für Erweiterung und Nachbarschafts- } \\
\text { politik Johannes Hahn an, es sei wichtig, dass die Wahlen in einer friedlichen Atmosphäre abgelaufen sind. } \\
\text { Allerdings sollte Belarus das Wahlsystem verbessern. }\end{array}$ \\
\hline 15.10 .2015 & $\begin{array}{l}\text { Der Sprecher des belarussischen Außenministeriums Dzmitry Mirontschyk kündigt während einer Pressekon- } \\
\text { ferenz an, Belarus sei offen für die Weiterführung des Dialogs mit der OSZE sowie mit dem Rat der EU zur } \\
\text { Wahlproblematik. Mirontschyk erklärt, dass Belarus eine vollständige Aufhebung der EU-Sanktionen erwartet. }\end{array}$ \\
\hline 15.10 .2015 & $\begin{array}{l}\text { Der Leiter für Osteuropa und Kaukasus der Europäischen Bank für Wiederaufbau und Entwicklung (EBRD) } \\
\text { Francis Malizha gibt bekannt, die EBRD sei an einer Ausweitung der Zusammenarbeit mit Belarus interessiert. }\end{array}$ \\
\hline 15.-16.10.2015 & $\begin{array}{l}\text { Präsident Lukaschenka hält sich in Burabai, Kasachstan, auf, um am Gipfeltreffen der GUS-Staatschefs sowie } \\
\text { des Obersten Wirtschaftsrates der Eurasischen Wirtschaftsunion teilzunehmen. }\end{array}$ \\
\hline 16.10 .2015 & $\begin{array}{l}\text { Nach Angaben der Zentralen Wahlkommission liegt die Wahlbeteiligung bei den Präsidentschaftswahlen } \\
2015 \text { in Belarus bei 87,2\%. Der amtierende Präsident Aljaksandr Lukaschenka erhielt 83,5\% aller Stimmen; } \\
\text { Taziana Karatkewitsch -4,4\%, Sjarhej Hajdukewitsch -3,3\% und Mikalaj Ulahowitsch - 1,7\%. }\end{array}$ \\
\hline 16.10 .2015 & $\begin{array}{l}\text { Die Zentrale Wahlkommission lehnt den Antrag von Taziana Karatkewitsch ab, die Ergebnisse der Präsident- } \\
\text { schaftswahlen für ungültig zu erklären. }\end{array}$ \\
\hline 16.10 .2015 & $\begin{array}{l}\text { Vertreter mehrerer Oppositionsparteien und Organisationen in Belarus schlagen dem Westen in einer gemein- } \\
\text { samen Erklärung vor, die Frage der vollständigen Aufhebung von Sanktionen gegen Belarus mit der Refor- } \\
\text { mierung des Wahlgesetzes und der Durchführung von freien und fairen Parlamentswahlen im Jahr } 2016 \text { zu } \\
\text { verknüpfen. }\end{array}$ \\
\hline 19.-27.10.2015 & $\begin{array}{l}\text { Die ehemalige Präsidentschaftskandidatin Taziana Karatkewitsch und der Leiter der Kampagne "Sag die } \\
\text { Wahrheit« Andrej Dzmitryeu unternehmen eine Reise nach Brüssel und Washington und treffen sich u. a. } \\
\text { mit folgenden Personen, um die Beziehungen zwischen Belarus und dem Westen zu besprechen: dem Leiter } \\
\text { der Delegation des Europäischen Parlaments für Beziehungen zu Belarus Bogdan Zdrojewski, dem Leiter der } \\
\text { Abteilung für bilaterale Beziehungen mit den Ländern der Östlichen Partnerschaft des Europäischen Auswär- } \\
\text { tigen Diensts Dirk Schübel und Vertretern des US-Außenministeriums. }\end{array}$ \\
\hline 23.10 .2015 & $\begin{array}{l}\text { Der stellvertretende Vorsitzende der Bewegung "Für die Freiheit«Jury Hubarewitsch sowie der Vorsitzender der } \\
\text { Partei BNF Aljaksej Janukewitsch werden von einem Minsker Gericht wegen ihrer Teilnahme an einer nicht } \\
\text { genehmigten Kundgebung am 4. Oktober gegen die Einrichtung eines russischen Luftwaffenstützpunktes in } \\
\text { Belarus zu einer Strafe von jeweils 3,6 Mio. BYR (umgerechnet knapp } 190 \text { Euro) verurteilt. }\end{array}$ \\
\hline
\end{tabular}




\begin{tabular}{|c|c|}
\hline 27.10 .2015 & $\begin{array}{l}\text { Außenminister Wladimir Makej kündigt an, Belarus unterstütze die Militärangriffe Russlands gegen den } \\
\text { sogenannten Islamischen Staat (IS) in Syrien. Die Aktion gegen Terroristen in Syrien widerspreche nicht dem } \\
\text { Völkerrecht, so Makej. }\end{array}$ \\
\hline 27.10 .2015 & $\begin{array}{l}\text { Die trilaterale Kontaktgruppe zur Beilegung der Krise in der Ukraine tagt erneut in Minsk. Die Seiten eini- } \\
\text { gen sich auf die Freilassung von Gefangenen, so der OSZE-Sonderbeauftragte für die Ukraine Martin Sajdik. }\end{array}$ \\
\hline 28.10 .2015 & $\begin{array}{l}\text { Freedom House bezeichnet Belarus im neuen Jahresbericht über Internetfreiheit (Freedom on the Net) für } \\
\text { den Zeitraum Juni } 2014 \text { bis Mai } 2015 \text { erneut als nicht frei. Folgende problematische Aspekte werden u. a. } \\
\text { im Bericht aufgelistet: Blockierung unabhängiger Internetportale und Druck auf freiberufliche Journalisten. }\end{array}$ \\
\hline 28.10 .2015 & $\begin{array}{l}\text { Außenminister Uladsimir Makej gibt in einem Interview für die russische Zeitung "Kommersant» bekannt, } \\
\text { dass eine Normalisierung der Beziehungen zwischen Belarus und der EU keine Auswirkungen auf die bela- } \\
\text { russisch-russischen Beziehungen haben wird. }\end{array}$ \\
\hline 29.10 .2105 & $\begin{array}{l}\text { Der Sonderberichterstatter für Belarus des UN-Menschenrechtsrats Miklós Haraszti stellt seinen Bericht über } \\
\text { die Meinungsfreiheit in Belarus während der UN-Generalversammlung vor. Die Unabhängigkeit der Medien in } \\
\text { Belarus sei u. a. wegen eines Genehmigungsprinzips für die Registrierung der Medien unmöglich, so Haraszti. }\end{array}$ \\
\hline 29.10 .2105 & $\begin{array}{l}\text { Der Rat der EU beschließt, Einreiseverbote und Kontensperrungen gegen } 171 \text { Personen, inklusive Präsident } \\
\text { Lukaschenka, sowie zehn Unternehmen in Belarus für vier Monate (31. Oktober bis zum 29. Februar 2016) } \\
\text { zu verlängern, jedoch sofort auszusetzen. Der Beschluss ist eine Reaktion auf die Freilassung aller politischen } \\
\text { Gefangenen in Belarus im August } 2015 \text { sowie auf den friedlichen Verlauf der Präsidentschaftswahlen. Belarus } \\
\text { rechne allerdings mit der schnellstmöglichen Aufhebung aller Sanktionen, so der Pressesprecher des Außen- } \\
\text { ministerium Dzmitry Mirontschyk. }\end{array}$ \\
\hline 29.10 .2105 & $\begin{array}{l}\text { Die USA verlängern ihre } 2006 \text { gegen neun belarussische Unternehmen verhängten Sanktionen bis zum April } \\
\text { 2016, jedoch mildern sie die restriktiven Maßnahmen ab. Die Sanktionen sehen die Vermögenssperrung der } \\
\text { betroffenen Unternehmen vor, allerdings sind Transaktionen mit den Unternehmen erlaubt. Der Pressesprecher } \\
\text { des belarussischen Außenministeriums Dzmitry Mirontschyk kündigt am kommenden Tag an, die vollständige } \\
\text { Aufhebung der Sanktionen werde zur Verbesserung der Beziehungen zwischen Belarus und den USA beitragen. }\end{array}$ \\
\hline 29.10 .2105 & $\begin{array}{l}\text { In Minsk nehmen ca. } 120 \text { Menschen an einer Kundgebung in Erinnerung an die Opfer der stalinistischen } \\
\text { Repressionen teil. }\end{array}$ \\
\hline 01.11 .2015 & $\begin{array}{l}\text { Anlässlich des Totengedenktags »Dsjady« organisiert die Belarussische Konservative Partei (BNF) mit Geneh- } \\
\text { migung der Stadt Minsk einen Gedenkzug, der mit einer Kundgebung in Kurapaty endet. Ca. } 400 \text { Menschen } \\
\text { nehmen an der Kundgebung teil. } 1988 \text { hatte der BNF-Gründer Sjanon Pasnjak erstmals die stalinistischen } \\
\text { Massenerschießungen, die während der 1930er Jahre in Kurapaty stattfanden, öffentlich gemacht. Seitdem } \\
\text { finden jährlich im November Veranstaltungen in Kurapaty statt. }\end{array}$ \\
\hline 04.11 .2015 & $\begin{array}{l}\text { Präsident Lukaschenka unterzeichnet einen Erlass zur Neudenominierung des belarussischen Rubels, die am } \\
\text { 1. Juli } 2016 \text { im Verhältnis von 1:10.000 durchgeführt wird. }\end{array}$ \\
\hline 04.-05.11.2015 & $\begin{array}{l}\text { Die stellvertretende Assistentin des US-Außenministers für Europa und Eurasien Bridget Brink und der stell- } \\
\text { vertretende Assistent des US-Außenministers für Demokratie, Menschenrechte und Arbeit Robert Berschin- } \\
\text { ski halten sich zu einem offiziellen Besuch in Minsk auf und führen Gespräche u. a. mit Oppositionsvertre- } \\
\text { tern, mit Menschenrechtlern sowie mit Außenminister Uladsimir Makej. Die USA seien bereit, weitere positive } \\
\text { Schritte in Bezug auf Belarus zu unternehmen, allerdings seien die Menschenrechtssituation und die demo- } \\
\text { kratische Entwicklung in Belarus dafür entscheidend, so Berschinski. }\end{array}$ \\
\hline 06.11 .2015 & $\begin{array}{l}\text { In Minsk findet die Amtseinführung des wiedergewählten Präsidenten Lukaschenka statt. In seiner Rede gibt } \\
\text { der Präsident bekannt, er sehe keinen Sinn in der Reformierung der existierenden Agrar- und Industriepo- } \\
\text { litik. Bezüglich der Außenpolitik werde die Schaffung des Unionsstaates mit dem »brüderlichen Russland« } \\
\text { fortgesetzt, gleichzeitig sei Belarus auch offen für eine gleichberechtigte Zusammenarbeit mit dem Westen, } \\
\text { so Lukaschenka. }\end{array}$ \\
\hline 09.-11.11.2015 & $\begin{array}{l}\text { Der belarussische Ministerpräsident Andrej Kabjakou hält sich zu einem offiziellen Besuch in Pakistan auf. } \\
\text { Er nimmt am 3. Belarussisch-Pakistanischen Businessforum teil und trifft sich u. a. mit dem Ministerpräsi- } \\
\text { denten Pakistans Nawaz Sharif. Das Treffen mündet in der Unterzeichnung eines Fahrplans für die Zusam- } \\
\text { menarbeit zwischen beiden Staaten für die Zeitperiode } 2016 \text { bis } 2020 \text {. Drüber hinaus wird ein Zentrum für } \\
\text { belarussische Kultur in der Nationalbibliothek Pakistans eröffnet. }\end{array}$ \\
\hline 09.-20.11.2015 & $\begin{array}{l}\text { Eine Expertenmission des Internationales Währungsfonds (IWF) unter Leitung von Peter Dolman, Leiter der } \\
\text { IWF-Mission in Belarus, hält sich in Belarus auf, um die Möglichkeit eines neuen dreijährigen Kreditpro- } \\
\text { gramms in Höhe von } 3 \text { Mrd. US-Dollar für Belarus zu besprechen. Allerdings werden keine Vereinbarungen } \\
\text { im Anschluss an die Mission getroffen. }\end{array}$ \\
\hline
\end{tabular}




\begin{tabular}{|c|c|}
\hline 10.11 .2015 & $\begin{array}{l}\text { Der Vorsitzender des Deutsch-Belarussischen Wirtschaftsclubs Hovsep Voskanyan erklärt während einer } \\
\text { Konferenz, die sich dem } 8 \text {. Tag der Deutschen Wirtschaft in Belarus (veranstaltet am 12.11.2015) widmet, } \\
\text { dass Deutschland an einer Mitgliedschaft von Belarus in der Eurasischen Wirtschaftsunion interessiert ist - } \\
\text { dadurch könnte Belarus »zur westlichen Tür in den europäischen Markt« werden. }\end{array}$ \\
\hline 11.-12.11.2015 & $\begin{array}{l}\text { Ministerpräsident Andrej Kabjakou besucht die Türkei. Er nimmt am Belarussischen Investitionsforum teil } \\
\text { und führt Gespräche u. a. mit dem türkischen Präsidenten Recep Tayyip Erdoğan sowie mit Spitzenmana- } \\
\text { gern türkischer Unternehmen. }\end{array}$ \\
\hline 11.-13.11.2015 & $\begin{array}{l}\text { In Minsk findet eine Konferenz zum Thema eingefrorener Konflikte im postsowjetischen Raum im Rahmen } \\
\text { der Initiative »Minsker Dialog« statt. An der vom Liberal Club organisierten Konferenz nehmen ca. } 30 \text { Experte } \\
\text { aus den ehemaligen sowjetischen Staaten, der EU und den USA teil. }\end{array}$ \\
\hline 12.11 .2015 & $\begin{array}{l}\text { Der Außenminister Litauens Linas Linkevicius trifft sich im Rahmen eines Arbeitsbesuchs in Belarus mit sei- } \\
\text { nem belarussischen Kollegen Uladsimir Makej. Die Aussetzung der EU-Sanktionen gegen Belarus sei eine } \\
\text { Chance zur Stärkung von positiven Beziehungen zwischen Belarus und der EU, so Linkevicius. }\end{array}$ \\
\hline 15.11 .2015 & $\begin{array}{l}\text { Verstärkte militärische Aktivitäten der NATO in der Nähe der belarussischen Grenzen seien als militärische } \\
\text { Bedrohung anzusehen, so der belarussische Verteidigungsminister Andrej Raukou. }\end{array}$ \\
\hline 17.11.2015 & $\begin{array}{l}\text { Die trilaterale Kontaktgruppe zur Beilegung der Krise in der Ukraine tagt erneut in Minsk. Alle betroffenen } \\
\text { Parteien sind dazu aufgefordert, die Verletzungen der Minsker Vereinbarungen sofort zu beenden, insbeson- } \\
\text { dere bezüglich der Waffenruhe und der Sicherheit an der faktischen Konfliktlinie, so der OSZE-Sonderbe- } \\
\text { auftragte für die Ukraine Martin Sajdik. }\end{array}$ \\
\hline 17.-18.11.2015 & $\begin{array}{l}\text { Außenminister Uladsimir Makej hält sich zu einem offiziellen Besuch in Deutschland auf und trifft sich u. a. } \\
\text { mit seinem deutschen Amtskollegen Frank-Walter Steinmeier, dem außen- und sicherheitspolitischen Bera- } \\
\text { ter der Bundeskanzlerin Christoph Heusgen, dem Vorsitzenden der Deutsch-Belarussischen-Parlamentarier- } \\
\text { gruppe im Deutschen Bundestag Oliver Kaczmarek sowie mit Mitgliedern dieser Gruppe. Es gebe eine reale } \\
\text { Perspektive einer schrittweisen Annäherung zwischen Belarus und dem Westen, so Steinmeier. }\end{array}$ \\
\hline 17.-20.11.2015 & $\begin{array}{l}\text { Die stellvertretende Außenministerin Aljona Kuptschyna hält sich zu einem offiziellen Besuch in Slowenien } \\
\text { auf. Sie führt Gespräche zu belarussisch-slowenischen Kooperationen und zur Entwicklung des Dialogs Bela- } \\
\text { rus-EU. Dazu trifft sie sich u. a. mit dem slowenischen Außenminister Karl Erjavec und nimmt an der } 17 . \\
\text { internationalen Business-Konferenz in Portorož teil. }\end{array}$ \\
\hline 18.11.2015 & $\begin{array}{l}\text { Der deutsche Botschafter in Belarus Peter Dettmar sieht im Einfrieren der EU-Sanktionen gegen belarussi- } \\
\text { sche Amtsträger und eine Reihe von Unternehmen eine gute Möglichkeit, die Belarus-EU Beziehungen neu } \\
\text { anzufangen. }\end{array}$ \\
\hline 18.-20. 11.2015 & $\begin{array}{l}\text { Der Präsident Serbiens Tomislav Nikolić hält sich zu einem offiziellen Besuch in Belarus auf und trifft sich } \\
\text { u. a. mit seinem belarussischen Amtskollegen Aljaksandr Lukaschenka. Nikolić hofft auf einen EU-Beschluss } \\
\text { zur Aussetzung der Sanktionen gegen Belarus. Die wirtschaftliche Zusammenarbeit zwischen Belarus und } \\
\text { Serbien müsse so stark wie die Freundschaft zwischen den beiden Ländern sein, so Nikolić. }\end{array}$ \\
\hline 19.11.2015 & $\begin{array}{l}\text { Das Gesellschaftliche Bologna Komitee, eine belarussische Organisation, erklärt, dass in Belarus der Druck } \\
\text { auf akademische Freiheiten und auf die Rechte der akademischen Gemeinschaft erhöht wird. }\end{array}$ \\
\hline 20.11 .2015 & $\begin{array}{l}\text { Iwan Kulesch wird von einem Hornauer Gericht wegen des Mords an drei Verkäuferinnen sowie wegen Dieb- } \\
\text { stahl und Raub zur Todesstrafe verurteilt. Der Europarat bedauert zutiefst das bereits zweite Todesurteil in } \\
\text { Belarus im Jahr 2015. }\end{array}$ \\
\hline 22.11 .2015 & $\begin{array}{l}\text { Die European Students' Union (ESU) ruft in einer Erklärung die belarussischen Behörden sowie die Verwal- } \\
\text { tung der belarussischen Hochschulen dazu auf, den Druck auf Studierende, die ihre akademischen Freiheiten } \\
\text { verteidigen, zu stoppen. Es geht um Studierende der Belarussischen Staatlichen Universität, die eine Erklä- } \\
\text { rung gegen die Einführung eines Gebührensystems für die Wiederholung von Prüfungen unterzeichnet hatten. }\end{array}$ \\
\hline 23.11 .2015 & $\begin{array}{l}\text { Der Ausschuss der UN-Generalversammlung für soziale, humanitäre und kulturelle Angelegenheiten nimmt } \\
\text { die von Belarus initiierte Resolution »Verbesserung der Koordinierung der Anstrengungen gegen Menschen- } \\
\text { handel« an. }\end{array}$ \\
\hline 24.11 .2015 & $\begin{array}{l}\text { In Minsk findet eine durch die Opposition organisierte Kundgebung in Erinnerung an das von A. Luka- } \\
\text { schenka initiierte Referendum im Jahr } 1996 \text { statt. Das Referendum erweiterte die Vollmachten des Präsiden- } \\
\text { ten erheblich; die Ergebnisse des Referendums wurden von der EU, der OSZE und den USA nicht anerkannt. }\end{array}$ \\
\hline 25.11 .2015 & $\begin{array}{l}\text { Der Abschuss eines russischen Militärflugzeugs durch die türkische Luftwaffe am } 24 \text {. November verursache } \\
\text { tiefes Bedauern und extreme Sorge, erklärt der Pressesprecher des belarussischen Außenministeriums Dzmi- } \\
\text { try Mirontschyk. }\end{array}$ \\
\hline
\end{tabular}




\begin{tabular}{|l|l|}
\hline 25.11.2015 & Das Abkommen über visafreien Reiseverkehr für touristische Zwecke zwischen Belarus und Israel trifft in Kraft. \\
\hline 26.11.2015 & $\begin{array}{l}\text { Die stellvertretende Außenministerin Aljona Kuptschyna beteiligt sich an der 6. Runde der inoffiziellen Minis- } \\
\text { tertreffen der Mitgliedsstaaten der »Östlichen Partnerschaft« in Tiflis. Kuptschyna plädiert u. a. für einen dif- } \\
\text { ferenzierten Ansatz im Rahmen der Östlichen Partnerschaft sowie für die Entwicklung eines Dialogs zwi- } \\
\text { schen der Eurasischen Wirtschaftsunion und der EU. }\end{array}$ \\
\hline 27.11.2015 & $\begin{array}{l}\text { Der Bericht der Leiter der Beobachtermission der Parlamentarischen Versammlung des Europarates Reha } \\
\text { Denemeç wird veröffentlicht. Der Bericht empfiehlt Belarus eine deutliche Verbesserung des Verfahrens zur } \\
\text { Stimmauszählung sowie der Regeln für Wahlbeobachter. }\end{array}$ \\
\hline 27.-28.11.2015 & $\begin{array}{l}\text { Der Präsident Aserbaidschans Ilham Alijew hält sich zu einem offiziellen Besuch in Belarus auf, wo er u. a. } \\
\text { Präsident Lukaschenka trifft. Im Anschluss an den Besuch werden eine Deklaration über die Entwicklung } \\
\text { des politischen Dialogs sowie ein Abkommen über Zusammenarbeit in wirtschaftlichen und sozialen Fra- } \\
\text { gen unterzeichnet. }\end{array}$ \\
\hline
\end{tabular}

Zusammengestellt auf der Grundlage der Meldungen der Nachrichten-Agentur BelaPAN und der Homepage naviny.by.

Sie können die gesamte Chronik ab dem 14.03.2011 auch auf<http://www.laender-analysen.de/belarus/> unter dem Link »Chronik« lesen.

Herausgeber: Forschungsstelle Osteuropa an der Universität Bremen, Deutsche Gesellschaft für Osteuropakunde e.V. und Internationales Bildungs- und Begegnungswerk, Dortmund

Die Meinungen, die in den Belarus-Analysen geäußert werden, geben ausschließlich die Auffassung der Autoren wieder. Abdruck und sonstige publizistische Nutzung sind nach Rücksprache mit der Redaktion gestattet. Redaktion: Heiko Pleines (verantwortlich), Olga Dryndova

$$
\text { Satz: Matthias Neumann }
$$

Belarus-Analysen-Layout: Cengiz Kibaroglu, Matthias Neumann und Michael Clemens

Alle Ausgaben der Belarus-Analysen sind mit Themen- und Autorenindex archiviert unter <www.laender-analysen.de> ISSN 2192-1350 @ 2015 by Forschungsstelle Osteuropa, Bremen 


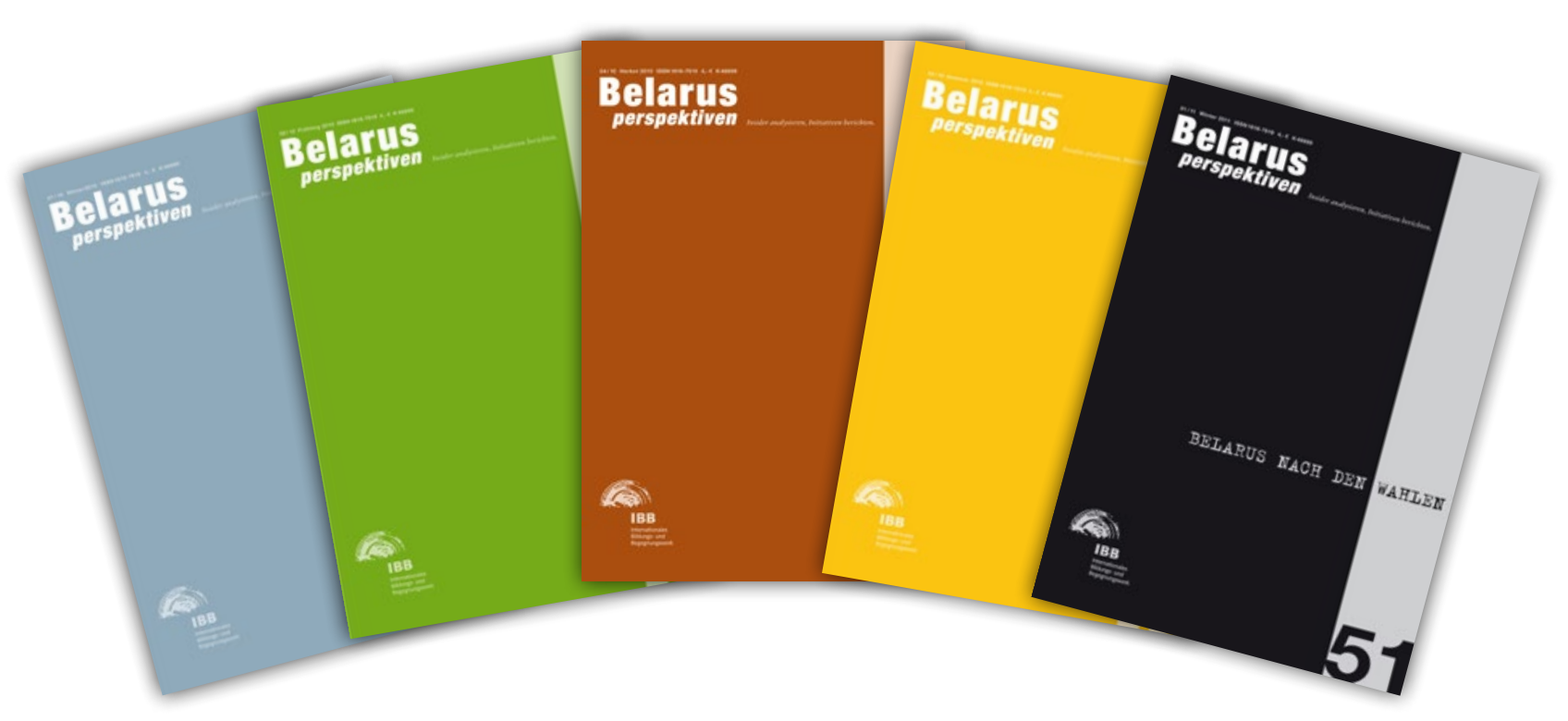

\section{Belarus}

\section{Perspektiven}

- Seit mehr als zehn Jahren berichten die Belarus Perspektiven aus einem fast unbekannten Land. Uns lesen Initiativen und Politiker, Journalisten und Unternehmer - all jene, die in Belarus etwas bewegen wollen.

- Durch unsere kritischen Berichte und Analysen sind unsere Leser nicht nur auf dem neuesten Stand sie erfahren auch, was hinter den Kulissen geschieht.

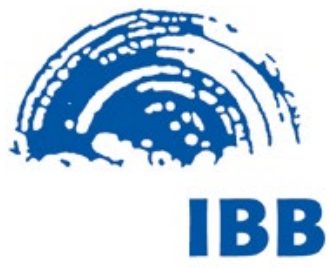

Internationales Bildungs- und Begegnungswerk
- Autoren der Belarus Perspektiven sind namhafte belarussische Journalisten, unabhängige Medienvertreter, Wirtschaftsexperten und zivilgesellschaftliche Akteure.

- Die Zeitschrift erscheint vierteljährlich und kostet inklusive Versand $15,-€$ im Jahresabonnement.

- Zu beziehen sind die Belarus Perspektiven beim IBB in Dortmund bzw. online unter <http://www.ibb-d.de/publikation-anfordernhtml.html>

- Ältere Ausgaben der Belarus Perspektiven finden Sie im Archiv als pdf-Datei zum Download, <http://www.ibb-d.de/bp-archiv.html> 


\section{Kostenlose E-Mail-Dienste der Forschungsstelle Osteuropa und ihrer Partner auf www.laender-analysen.de}

Die Länder-Analysen bieten regelmäßig im kostenlosen Abonnement kompetente Einschätzungen aktueller politischer, wirtschaftlicher, sozialer und kultureller Entwicklungen in Ostmitteleuropa und der GUS. Sie machen das Wissen, über das die wissenschaftliche Forschung in reichem Maße verfügt, für Politik, Wirtschaft, Medien und die interessierte Öffentlichkeit verfügbar. Autoren sind internationale Fachwissenschaftler und Experten.

Die einzelnen Länder-Analysen werden von der Forschungsstelle Osteuropa an der Universität Bremen und der Deutschen Gesellschaft für Osteuropakunde jeweils mit unterschiedlichen Partnern und Sponsoren herausgegeben. Die Redaktionen der Länder-Analysen bestehen aus Wissenschaftlern mit langjähriger Forschungserfahrung.

Die Länder-Analysen bieten regelmäßig Kurzanalysen zu aktuellen Themen, ergänzt um Grafiken und Tabellen sowie Dokumentationen. Zusätzlich gibt es eine Chronik aktueller Ereignisse. Alle Länder-Analysen sind auch mit Archiv und Indizes online verfügbar unter $<$ www.laender-analysen.de $>$.

\section{Belarus-Analysen}

Erscheinungsweise: zweimonatlich

Abonnement unter: <http://www.laender-analysen.de/belarus/>

\section{Caucasus Analytical Digest}

In englischer Sprache. Erscheinungsweise: monatlich

Abonnement unter: <http://www.css.ethz.ch/publications/newsletter_CAD_EN>

\section{Polen-Analysen}

Erscheinungsweise: zweimal monatlich

Abonnement unter: <http://www.deutsches-polen-institut.de/newsletter/polen-analysen/>

\section{Russland-Analysen}

Erscheinungsweise: zweiwöchentlich

Abonnement unter: <http://www.laender-analysen.de/russland/>

\section{Russian Analytical Digest}

In englischer Sprache. Erscheinungsweise: zweimal monatlich

Abonnement unter: <http://www.css.ethz.ch/publications/newsletter_RAD_EN>

\section{Ukraine-Analysen}

Erscheinungsweise: zweimal monatlich

Abonnement unter: <http://www.laender-analysen.de/ukraine/>

\section{Zentralasien-Analysen}

Erscheinungsweise: monatlich

Abonnement unter: <http://www.laender-analysen.de/zentralasien/>

\section{Bibliographische Dienste}

Die Bibliographien informieren über englisch- und deutschsprachige Neuerscheinungen zu Belarus, Russland, Ukraine sowie zu den zentralasiatischen und kaukasischen Staaten. Erfasst werden jeweils die Themenbereiche Politik, Außenpolitik, Wirtschaft und Soziales.

Erscheinungsweise: viermal jährlich

Abonnement unter: <http://www.laender-analysen.de/bibliographies/belarus.php $>,<$ http://www.laender-analysen. de/bibliographies/russia.php>, <http://www.laender-analysen.de/bibliographies/ukraine.php>, <http://www.laenderanalysen.de/bibliographies/caucasus_ca.php> 